
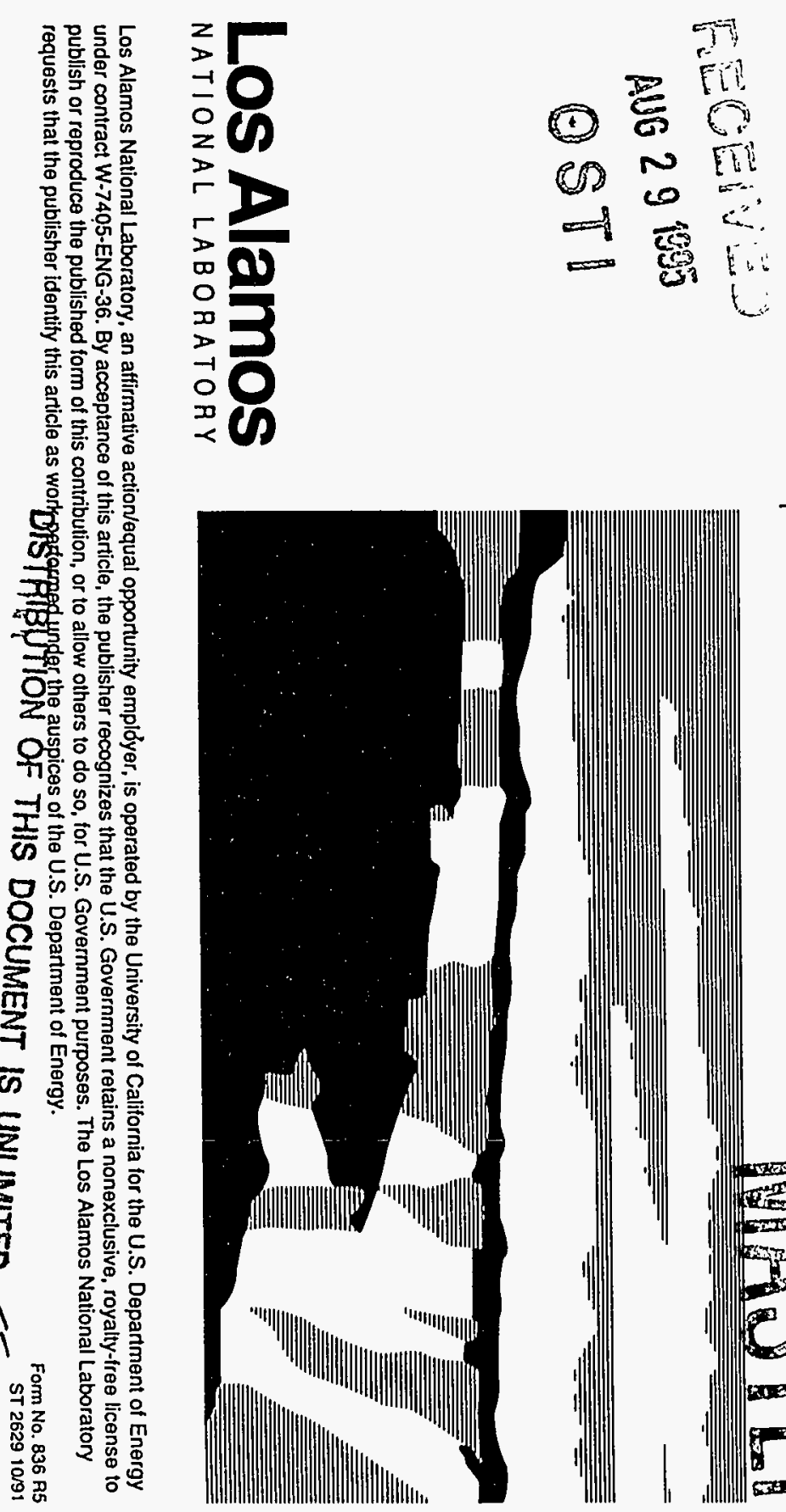

This report was prepared as an account of work sponsored by an agency of the United States Government. Neither the United States Government nor any agency thereof, nor any of their employees, makes any warranty, express or implied, or assumes any legal liability or responsibility for the accuracy, completeness, or usefulness of any information, apparatus, product, or process disclosed, or represents that its use would not infringe privately owned rights. Reference herein to any specific commercial product, process, or service by trade name, trademark, manufacturer, or otherwise does not necessarily constitute or imply its endorsement, recommendation, or favoring by the United States Government or any agency thereof. The views and opinions of authors expressed herein do not necessarily state or reflect those of the United States Government or any agency thereof.
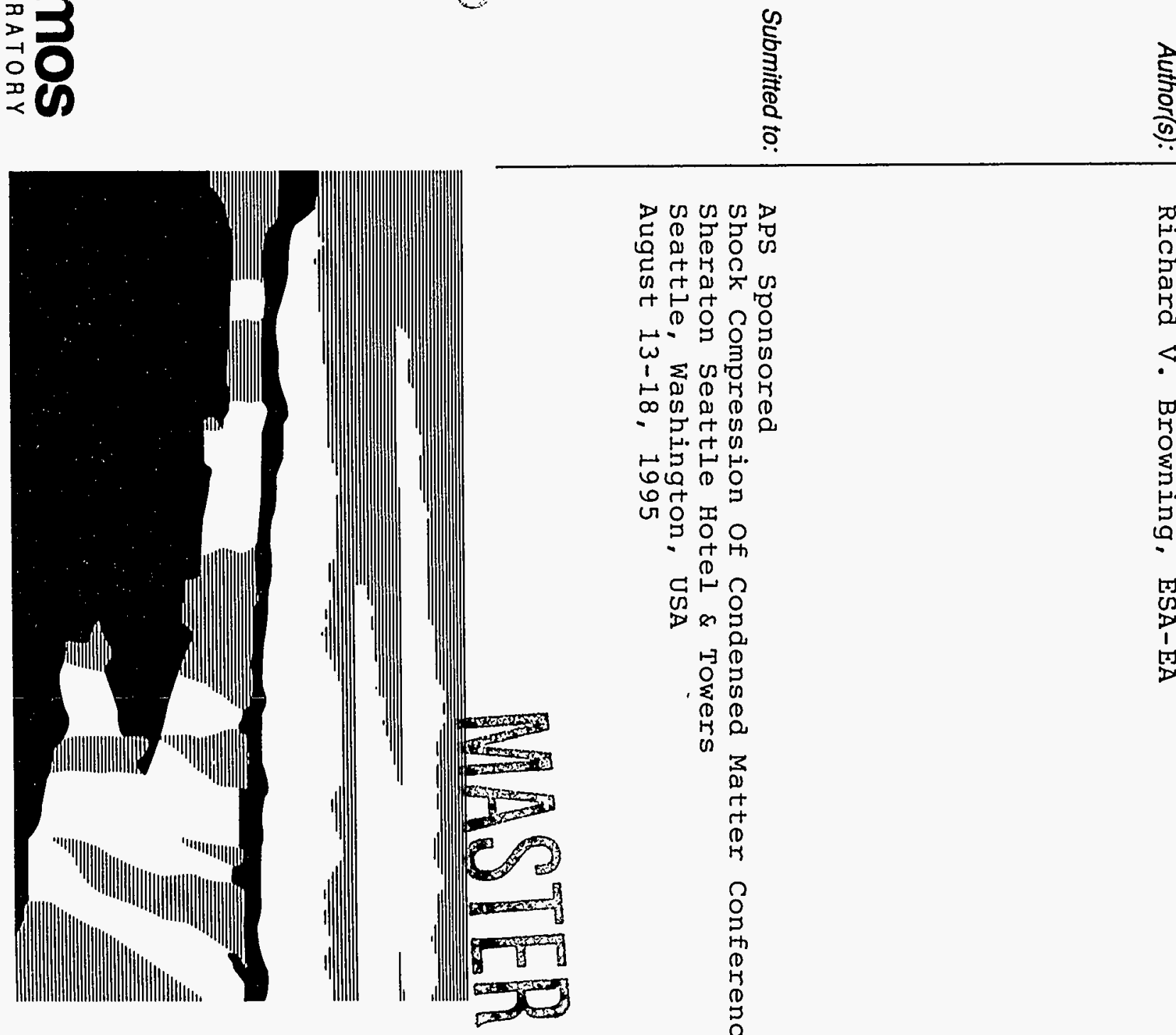


\section{DISCLAIMER}

Portions of this document may be illegible in electronic image products. Images are produced from the best available original document. 


\title{
MICROSTRUCTURAL MODEL OF MECHANICAL INITIATION OF ENERGETIC MATERIALS
}

\author{
Richard V. Browning \\ Los Alamos National Laboratory \\ PO Box 1663 \\ Los Alamos, NM 87545
}

\begin{abstract}
Mechanical initiation of chemical reactions in energetic materials depends on microstructural details of the materials. Several models are described in the literature that are appropriate for a continuum, such as energy dissipation from plastic flow, or shear bands. A technique is presented here for developing initiation models using relations between macroscopic variables and conditions at inter-grain contact areas in a granular material. The chemical processes that lead to initiation are included by using a 2-D numerical heat transfer model of energy flux on a surface spot of a half-space medium with multiple species chemical reactions. A number of calculations are done and the time to ignition at a given fluence is obtained as a function of spot size. Then simple kinematic relations between macroscopic stresses and the inter-grain contact forces are developed and the shearing velocity at the contact region is related to the macroscopic shear strain rate. Combining these relations leads to an ignition criterion in terms of macroscopic pressure, shear-strain rate and time. Even though very simple approximations for most relations are used, the overall result is similar to commonly used detonation initiation criterion. Experiments to define the constants in the model are under development and will be described.
\end{abstract}

\section{INTRODUCTION}

The initiation of reaction in energetic materials by mechanical impact is an important problem in terms of handling safety of propellants and explosives. The fundamental mechanism is evidently somewhat different compared with shock initiation. Initiation by a shock occurs within a few microseconds at initial pressure levels of around 20 kbar and above. A variety of models are available to model shock initiation, ranging from simple $p^{2} \tau$ models to complex numerical methods $(1,2)$. In a mechanical impact situation, initiation can be achieved at very low pressure levels, 2-3 kbar, but the time to ignition is very long, hundreds of microseconds. The result is not a conventional detonation and detonation initiation models do not predict the reaction. The observed results range from blackened material, to partial reactions that appear to transition to a detonation, on up to very energetic reactions that are close to full detonations in their effects. Here we develop a micromechanical based model for initiation under long duration impact conditions. The goal is to capture plausible, but very simple, mechanics in a form that provides insight into the actual initiation phenomenology and results in a simple model.

Plastic bonded explosives and propellants are made of granular energetic materials, such as TATB or HMX. The energetic grains are separated by a film of binder. The thickness depends on the fraction of binder present. Under mechanical loading, some of the grains will touch and relative sliding will occur when subjected to shearing deformation. This relative motion at grain contact points dissipates energy and can result in thermal ignition in this highly localized region. The grain 
sizes in most materials are in the range of 10 to 200 $\mu \mathrm{m}$. The corresponding contact regions might be a few percent of that size.

The model that emerges needs verification or calibration against experiments. We conclude with a brief description of some in-progress experiments designed to provide the required information.

\section{THERMO-CHEMICAL MODELING}

The modeling is done using a multi-species, multi-reaction chemistry model incorporated into a finite element based heat transfer program. The code used is Chemical Topaz(3), and the model is one developed by McGuire and Tarver(4). Chemical Topaz is a finite element based 2-D heat transfer program with provision for multi-species, multi-reaction chemical processes as described by Nichols(5).

The conceptual model is a uniform thermal flux over a spot or strip on a half space of material. The flux originates as mechanical work, but to the heat transfer problem this is boundary condition. In general the interaction of a complex chemical reaction and a heat transfer problem with time dependent boundary conditions leads to a very complex system of equations. To develop some insight into the characteristics of the problem we need to simplify the problem sufficiently to arrive at an algebraic equation instead of a set of PDEs. The customary technique is to simplify the chemistry to a single Arrhenius reaction, use simple boundary conditions such as a constant flux, and seek an approximate analytical solution(6). In order to preserve the ability to use more interesting chemical models we numerically integrate the problem with constant boundary conditions. We apply a constant flux, $\phi$, over a given spot size and run the calculation until a reaction occurs. The product of the flux and the time to ignition, $t_{i g}$, gives the critical fluence, $\Phi_{i g}$. For a given spot size, the critical fluence is a function of the flux, or time. Figure 1 shows the results of a number of calculations run for differing spot sizes and fluencelevels plotted on logarithmic scales. Taking results for a given spot size and fitting a straight line results in a power law model that provides reasonable coverage for results for this particular chemical model and range of conditions. It turns out that the spacing for the different spot sizes is reasonably uniform so the overall model is a power law in both size and time. That is,

$$
\Phi_{i g}=0.0078 a\left(t_{i g} / a^{2}\right)^{0.7876},
$$

where $a$ is the spot diameter in $\mu \mathrm{m}$, time is in $\mu \mathrm{s}$, and the fluence is in $\mathrm{cal} / \mathrm{cm}^{2}$. This model assumes the flux is constant in time and uses the ODTX based chemistry model for HMX. In effect, the chemistry and heat transfer behavior is captured in the constants of this equation. Similar models could be developed for other chemistry and time history combinations, for reasonably broad ranges of conditions.

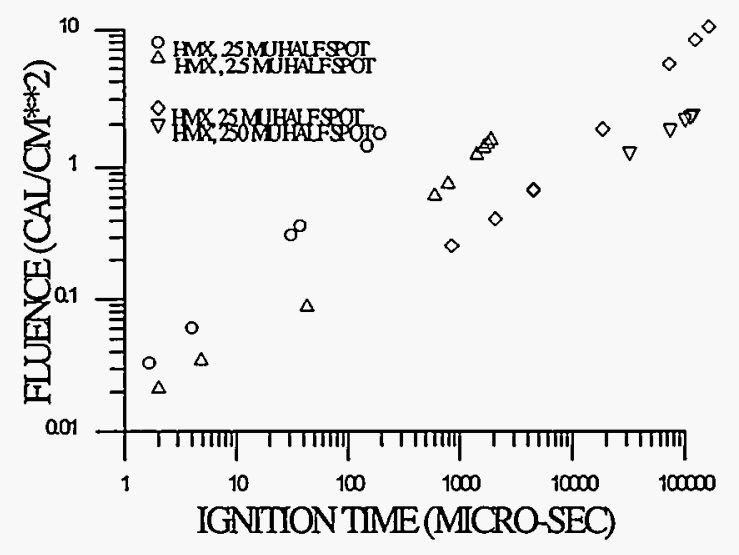

Figure 1. Fluence at ignition for $\mathrm{HMX}$ from Chemical TOPAZ calculations using ODTX model.

\section{MECHANICAL INTERFACE MODEL}

The interface conditions at a contact point between two grains is very complex in reality. The grains come in a variety of shapes, and while sharp facets are the preferred crystal growth mechanism, the mechanical damage incurred in fabrication processes result in irregular but rounded shapes. When pressed into a billet some fracturing and plastic flow of grains as well as flow of the binder occurs in the process of consolidating a molding 
powder of density 0.7 to a final density of 1.9 . This complex situation is not easily modeled in detail. There are two parts to the problem, estimating the contact area and modeling the (friction) forces that result from sliding motion.

The customary friction model used for large scale sliding situations, in some sense incorporates in a simple way the complex geometric interactions that occur at real interfaces. We adopt the standard friction model to develop a tangential force and combined with the expressions for sliding velocity will produce our mechanical work expression,

$$
\phi=\dot{\Phi}=\beta \sigma_{c} v,
$$

where $v$ is the sliding velocity.

The other part of the interface model involves calculating the contact area, given the force at an interface. This is done using the standard Hertz solution (7) for two spherical bodies in contact. In this case, we assume equal radii of curvature, but the differences are not profound if they are unequal. The standard solution gives the contact patch radius as,

$$
a=0.721 R \sqrt[3]{F_{R} K_{D} C_{E}}
$$

where $K_{D}=R, C_{E}=2\left(1-v^{2}\right) / E$. Here $E$ and $v$ are the elastic constants and $R$ is the sphere radius or our grain size. The normalized maximum contact stress is then expressed in terms of the contact force and patch radius as,

$$
\sigma_{C}=1.5 F_{R} / \pi a^{2}
$$

\section{COUPLING MACROSCOPIC STATE TO MICROSCOPIC STATE}

The macroscopic stress state and the strain rate must be coupled to the microscopic intergranular forces and sliding velocities. Again this is a very complex situation in reality, but we choose some simple approximations. To obtain the normal force estimates we employ a hexagonal array of close packed spheres. Considering a single sphere out of a horizontal layer, the sphere is supported on three spheres below, and loaded by three spheres from above. There are also six points of contact with adjacent spheres at the equator, but these carry no load in this case. The relation between the averaged vertical force on a single sphere and the three top or bottom loads is obtained from a trigonometric relation and is, $F_{R}=F_{A} / \sqrt{6}$. Dividing by the projected planar area occupied by a sphere, that is the enclosing hexagonal area, we obtain a relation between the macroscopic stress and the normal force at the contact point,

$$
F_{R}=\sqrt{2} \sigma_{A} R^{2}
$$

where $\sigma_{A}$ is the average global stress (or pressure) magnitude.

We also need a relation between the macroscopic shear strain rate, and the microscopic sliding velocity. If the grains can rotate, then this would be non-trivial, however we assume that the grains are geometrically constrained to not rotate. In this case a reasonable estimate is simply,

$$
v=R \dot{\gamma},
$$

where $\dot{\gamma}$ is the magnitude of the global shear strain rate. This is based on the notion of planes of grains sliding on one another.

The details of these relations are not critical to the outcome, as long as they are proportional relations.

\section{OVERALL MODEL}

Combining Equations 3, 4 and 5 we obtain,

$$
\frac{\sigma_{C}}{E}=\frac{0.938}{\left(2 \sqrt{2}\left(1-v^{2}\right)\right)^{2 / 3}}\left(\frac{\sigma_{A}}{E}\right)^{1 / 3}
$$

or for the specific case of $v=0.33$,

$$
\frac{\sigma_{C}}{E}=0.506\left(\frac{\sigma_{A}}{E}\right)^{1 / 3} \text {. }
$$

This is an interesting relation because small global stresses produce much higher local stresses. For example, if $\mathrm{E}=10000 \mathrm{MPa}$ and the global stress is 1 $\mathrm{MPa}$, then the contact stress is $95 \mathrm{MPa}$.

Next we use Equation 2 and 6 to obtain $\dot{\Phi}=\beta \sigma_{C} R \dot{\gamma}$, and then use Equation 8 integrated over time assuming a constant $\dot{\gamma}$ to obtain, 


$$
\Phi=0.506 \beta R E \dot{\gamma}\left(\frac{\sigma_{A}}{E}\right)^{1 / 3} t .
$$

Finally use Equation 1 to arrive at,

$$
C=\sigma_{A}{ }^{0.66} \dot{\gamma}^{1.27} t_{i g}^{0.27},
$$

where $C$ is a collection of material constants.

This equation assumes a square wave type pressure and shear-strain rate. If other wave shapes are used different exponents and constants would emerge, but the general form of the solution would remain.

\section{EXPERIMENTS}

To calibrate this type of model we need a series of experiments resulting in ignition, at a measured time, pressure, and shear strain rate. A major problem is measuring shear-strain rate. The time of ignition and pressure history are measured in a few experiments, but unfortunately in most safety oriented experiments none of the items are measured. A series of experiments patterned after those of Chidester(8) will provide the information needed to calibrate and verify this simple model. In these experiments a blunt impactor deforms a confined sample of material. The time to ignition can be altered by changing the shape, mass, and velocity of the impactor. Pressures are directly measured with embedded instrumentation. The shear strain-rate must be obtained from numerical calculations of the mechanical deformation. These can be cross checked against measured deformations of the outer shells and other experimental information. The experiments are in progress at the time of writing. Other potential experimental configurations include that of BoyleFrey-Blake(9)

\section{CONCLUSIONS}

The simple model developed here provides a quick, but approximate, method of assessing the likelihood of initiation in a mechanical impact situation. It is easily implemented in wave propagation codes or their post-processors. It still needs experimental calibration and verification.
More importantly it demonstrates a methodology for developing micro-mechanical based initiation models of a variety of levels for different purposes. Different mechanisms can be substituted for the simple friction laws used here, as one example. Another approach is to use a full numerical integration of the heat and chemistry equations at selected points in a numerical simulation of the deformation of a system. This avoids the problem of assuming a particular time history in the thermochemical computations. The problem of following the reaction once started is also an interesting, but much more challenging problem.

\section{ACKNOWLEDGMENTS}

I appreciate the help received from Albert Nichols, LLNL, in obtaining the Chemical-Topaz program. I also thank Philip Howe for providing technical guidance and encouragement while working on this project.

\section{REFERENCES}

1. Walker, F.E. and Wasley, R. J., Explosivestoffe, No. 1,9, 1969.

2. Mader, C.L., Numerical Modeling of Detonations, U. California Press, 1979, pp. 208-218.

3. Shapiro, A.B., TOPAZ2D-A Two-Dimensional Finite Element Code for Heat Transfer Analysis, UCID-20824, Lawrence Livermore National Laboratory, July 1986. 4. McGuire, R.R. and Tarver, C.M., "Chemical Decomposition Models for the Thermal Explosion of Confined HMX, TATB, RDX, and TNT", Seventh (International) Symposium on Detonation, June 1981, pp. 56-64.

5. Nichols, A.L. III, Chemical-TOPAZ - An Addendum to the TOPAZ manual, UCRL-ID-104558 Add. 1, Lawrence Livermore National Laboratory, Jan. 1993.

6. Frank-Kamenetskii, D.A., Diffusion and Heat Transfer in Chemical Kinetics, Plenum, New York, 1969, Ch. 7.

7. Roark, R.J. and Young, W.E., Formulas for Stress and Strain Fifth Ed., McGraw-Hill, New York, 1975, p. 516.

8. Chidester, S.K. and Green, L.G., A Frictional Work Predictive Method for the Initiation of Solid High Explosives from Low Pressure Inputs, Tenth (International) Symposium on Detonation, July 1993.

9. Boyle, V., Frey, R. and Blake, O., Combined Pressure Shear Ignition of Explosives, Ninth (International) Symposium on Detonation, Aug. 1989, pp. 3-17. 